\title{
THEORETICAL PROBLEMS IN THE INTERSTELLAR MEDIUM
}

\author{
J. MICHAEL SHULL \\ Joint Institute for Laboratory Astrophysics \\ University of Colorado and \\ National Institute of Standards \& Technology \\ Boulder, CO 80309-0440 USA
}

\begin{abstract}
I review recent theoretical developments in the structure, heating, and ionization of the interstellar medium. Among the unsolved issues are: the vertical support of the gas layer; the ionization source for the $\mathrm{H}^{+}$layer and diffuse line emission above the Galactic plane; the physics of stochastic heating of small dust grains and the resulting infrared spectra. Turbulent mixing may play an important role in the intercloud medium and diffuse emission above the disk. I conclude with some possible analogies between the intergalactic medium and the interstellar medium.
\end{abstract}

\section{INTRODUCTION}

In this brief review, I intend to summarize several theoretical problems dealing with the structure, ionization, and spectra of the interstellar medium (ISM). Because of restrictions of time and space, I will restrict this discussion primarily to issues of the diffuse ISM, although I will close with several thoughts on how studies of our Galactic ISM may be applicable to research on the intergalactic medium.

I have chosen four topics for this review: (1) the problem of support of the diffuse ISM above the Galactic disk; (2) the source of matter and ionization for the Galactic halo; (3) stochastic heating and infrared emission from dust grains; and (4) the structure, ionization, and cooling of intergalactic Ly $\alpha$ clouds.

\section{THE SUPPORT OF INTERSTELLAR MATTER ABOVE THE DISK}

Ultraviolet studies by the Copernicus and $I U E$ satellites have shown that, within several $\mathrm{kpc}$, the average gas distribution above the Galactic plane falls off with Gaussian scale heights ranging between 300 and $1000 \mathrm{pc}$. This range reflects the types of gas tracers (Bohlin, Savage, \& Drake 1979; Van Steenberg \& Shull 1988). In the IUE data, H I has a Gaussian scale height of $332 \mathrm{pc}$, similar to Si II (352 pc) and S II (320 pc), but in sharp contrast to Fe II (1110 pc). Edgar \& Savage (1989) also find large exponential scale heights for the refractory species Ca II $(h=1 \mathrm{kpc})$ and Ti II $(h \geq 2 \mathrm{kpc})$. The remarkable 
smoothness of the Ti II and Ca II distributions suggests a model in which strong mixing occurs. These data probably also require grain destruction in hot gas or shocks, perhaps in a Galactic "fountain" that mixes together cold clouds (with depleted abundances) and hot gas to form a warm, frothy intercloud medium. Recent studies at 21-cm and Ly $\alpha$ (Lockman 1984; Lockman, Hobbs, \& Shull 1986) identify a layer of warm H I (the "Lockman Layer") at similar large distances above the disk. A layer of $\mathrm{H}^{+}$at $z \approx 1 \mathrm{kpc}$ (the "Reynolds layer") has been identified in diffuse $\mathrm{H} \alpha$ emission and pulsar dispersion. An open question is whether the refractory-element scale heights and the ionized layer arise in a "Galactic fountain" in which ballistic motion provides some of the support against gravity.

The integrated column densities of $\mathrm{H} \mathrm{I}$ and $\mathrm{H}_{2}$ through the disk are approximately $N(\mathrm{H} \mathrm{I})=6.0 \times 10^{20} \mathrm{~cm}^{-2}\left(\Sigma(\mathrm{H} \mathrm{I})=4.8 \mathrm{M}_{\odot} \mathrm{pc}^{-2}\right)$ and $N\left(\mathrm{H}_{2}\right)=2.4 \times 10^{20} \mathrm{~cm}^{-2}\left(\Sigma\left(\mathrm{H}_{2}\right)=\right.$ $\left.1.9 M_{\odot} \mathrm{pc}^{-2}\right)$. The ionized hydrogen layer (Reynolds 1990 ) has $N(\mathrm{H} \mathrm{II})=2.4 \times 10^{20}$ $\mathrm{cm}^{-2}\left(\Sigma(\mathrm{H} \mathrm{I})=1.9 \mathrm{M}_{\odot} \mathrm{pc}^{-2}\right)$, some $23 \%$ of the gas mass. Thus, in all forms, the gaseous hydrogen surface density of the Galactic disk is $8.3 M_{\odot} \mathrm{pc}^{-2}$. Adding the associated helium ( $26 \%$ by mass) yields a total gas-phase surface density $\Sigma_{\text {gas }}=11.6 M_{\odot} \mathrm{pc}^{-2}$, a significant fraction of the "Oort limit", recently determined as either $(67 \pm 5) M_{\odot}$ pc $^{-2}$ (Bahcall 1984) or $(46 \pm 9) M_{\odot} \mathrm{pc}^{-2}$ (Kuijken \& Gilmore 1989). In the latter case, the interstellar matter in the disk provides $25 \%$ of the total binding mass. Thus, fluctuations in the interstellar gas column induced by star formation and wind/supernova activity could have important effects on stellar dynamics.

Cox (1990) has noted that hydrostatic support of this mass density requires a total pressure in the midplane of $P / k=n T \approx 25,000 \mathrm{~cm}^{-3} \mathrm{~K}$, much larger than the values of $3000-10,000 \mathrm{~cm}^{-3} \mathrm{~K}$ attributed to thermal pressure. It is argued that a mean magnetic field of $5 \mu \mathrm{G}$ in the disk would provide one-third of the required pressure $\left(B^{2} / 8 \pi k=7200\right.$ $\mathrm{cm}^{-3} \mathrm{~K}$ ), although the precise field-line geometry is unspecified. An analysis of pulse dispersion and Faraday rotation toward nearby pulsars (Lyne \& Smith 1989; Rand \& Kulkarni 1989) concludes that the random component of magnetic field is significant. Remaining to be determined are the length scale of the field fluctuations and a dynamical model of magnetic structure above the disk.

Another outstanding theoretical problem in interstellar astronomy is to understand the mechanism by which a Galactic fountain affects the "ballistics" of clouds above the disk, threaded by magnetic fields and pulled downward by gravity. The damping of MHD waves generated by the interaction of shock waves with these clouds may provide a significant heat source for the warm H I (Ferrière, Zweibel, \& Shull 1988). However, the vertical extent of the fields and the MHD waves is highly uncertain.

\section{THE IONIZATION OF DIFFUSE HALO GAS}

Diffuse $\mathrm{H} \alpha$ and UV line emission from gas in the low halo have been studied by Reynolds $(1982,1990)$ and by Martin \& Bowyer $(1990)$. The $\mathrm{H} \alpha$ fluxes, if they are produced by recombination of photoionized gas, require a Lyman-continuum photon flux $\Phi_{c}=4 \times 10^{6}$ $\mathrm{cm}^{-2} \mathrm{~s}^{-1}$ and a total power of $7 \times 10^{41} \mathrm{ergs} \mathrm{s}^{-1}$ over the entire disk, assuming 1 Rydberg per ionizing photon and an effective disk area of $870 \mathrm{kpc}^{-2}$. These requirements provide severe constraints on possible sources. For example, supernovae of $10^{51}$ erg exploding at a rate of 1 per 15 yrs (a 30-yr interval each for Types $\mathrm{I}$ and II) provide $2 \times 10^{42} \mathrm{ergs} \mathrm{s}^{-1}$, some 
fraction of which is converted to ionizing photons. One would need to tap some $35 \%$ of the SN power to explain the $\mathrm{H} \alpha$ background. From such reasoning, Reynolds (1982) suggested that $O$ stars are the only plausible candidate for the ionizing fluxes, with $(1-3) \times 10^{7}$ photons $\mathrm{cm}^{-2} \mathrm{~s}^{-1}$ in the Galactic disk, diminished by transport through the disk HI.

Another argument for diffuse photoionization has been advanced by Mathis (1986) to explain the observed intensity ratio $[\mathrm{S} \mathrm{II}] / \mathrm{H} \alpha \approx 0.5$. Diffuse Lyman continuum photons can be produced by recombination layers around $\mathrm{H}$ I clouds, but it is unclear whether they can get to the halo. The action of superbubbles breaking through portions of the disk H I has been suggested as a necessary condition for leaking $15 \%$ of the $\mathrm{O}$-star flux into the halo (Norman \& Panagia - see Norman 1990).

An alternate mechanism for explaining the diffuse emission lines from the "Reynolds layer" is that of Turbulent Mixing Layers. Created when cold gas is entrained by hot gas through Kelvin-Helmholtz or Rayleigh-Taylor instabilities, mixing layers (Begelman \& Fabian 1990) are likely to be ubiquitous throughout the violent interstellar medium, near supernova remnants and superbubbles, along "chimney walls" when superbubbles break through the H I layer, and near regions of star formation. Slavin, Shull, \& Begelman (1992) have calculated the time-dependent cooling and emission-line spectra that result in these layers. A characteristic intermediate temperature in the layer is:

$$
T_{m}=\frac{\dot{m}_{h} T_{h}+\dot{m}_{c} T_{c}}{\dot{m}_{h}+\dot{m}_{c}} \approx\left[\frac{\eta_{h}+\eta_{c}\left(T_{c} / T_{h}\right)^{1 / 2}}{\eta_{c}+\eta_{h}\left(T_{c} / T_{h}\right)^{1 / 2}}\right]\left(T_{c} T_{h}\right)^{1 / 2}=\xi\left(T_{h} T_{c}\right)^{1 / 2}
$$

where $T_{h}$ and $T_{c}$ are the temperatures of hot and cold gases, where $\dot{m}_{h}=\eta_{h} \rho_{h} v_{t}$ is the entrapment rate of hot gas per unit area, and $\dot{m}_{c}=\eta_{c}\left(\rho_{h} \rho_{c}\right)^{1 / 2} v_{t}$ is the cold gas entrapment rate. Here, $\rho_{h}$ and $\rho_{c}$ are the mass densities of hot and cold gas, and $v_{t}$ is the velocity of the hot gas penetrating the mixing layer. The rate of mixing is $\eta_{h} v_{t}$, and the overall parameter $\xi$ measures the mass-weighted rate of entrainment of the two species. The intermediate temperature $T_{m}$ is approximately the geometric mean of $T_{h}$ and $T_{c}$ and ranges between $10^{5.3} \mathrm{~K}$ and $10^{5.7} \mathrm{~K}$ in our models.

When hot and cold gas mix on timescales short compared with the cooling time, the intermediate-temperature gas radiates profusely in UV, EUV, and optical emission lines. The total energy radiated from the layer is balanced by the enthalpy flux of entrained gas $\left(5 P \eta_{h} v_{t} / 2\right)$, where $P$ is the gas pressure and $v_{t}$ is the velocity of hot gas. In addition to the collisionally excited line emission, EUV photons are reprocessed by photoionization into additional $\mathrm{H} \alpha$ and other optical lines. We find that for $\tilde{T}=10^{5.5} \mathrm{~K}$, the turbulent mixing has an efficiency of $8-10 \%$ for converting energy flux into $\mathrm{H} \alpha$ photons. The absolute fluxes from these layers are directly proportional to pressure. Mixing layers at pressure $P / k \approx 10^{4}$ $\mathrm{cm}^{-3} \mathrm{~K}$ could be responsible for about $10 \%$ of the high-latitude $\mathrm{H} \alpha$ background, but conld produce nearly all of the C IV emission (Martin \& Bowyer 1990), with proper ratios of [S II] $/ \mathrm{H} \alpha,[\mathrm{O}$ III] $/ \mathrm{H} \alpha$, and C IV $\lambda 1550 / \mathrm{O}$ III] $\lambda 1663$. The absolute intensities of C IV $\lambda 1550$ for mixing layers of pressure $n T=3000 \mathrm{~cm}^{-3} \mathrm{~K}$ are shown in Figure 1.

A related feature of these mixing layers is their predicted absorption column densities in the high ions, C IV, Si IV, N V, and O VI. The models that fit the C IV and O III] emission-line intensities appear to predict columns in reasonable agreement with UV observations (Jenkins 1978a,b; Savage \& Massa 1987). 


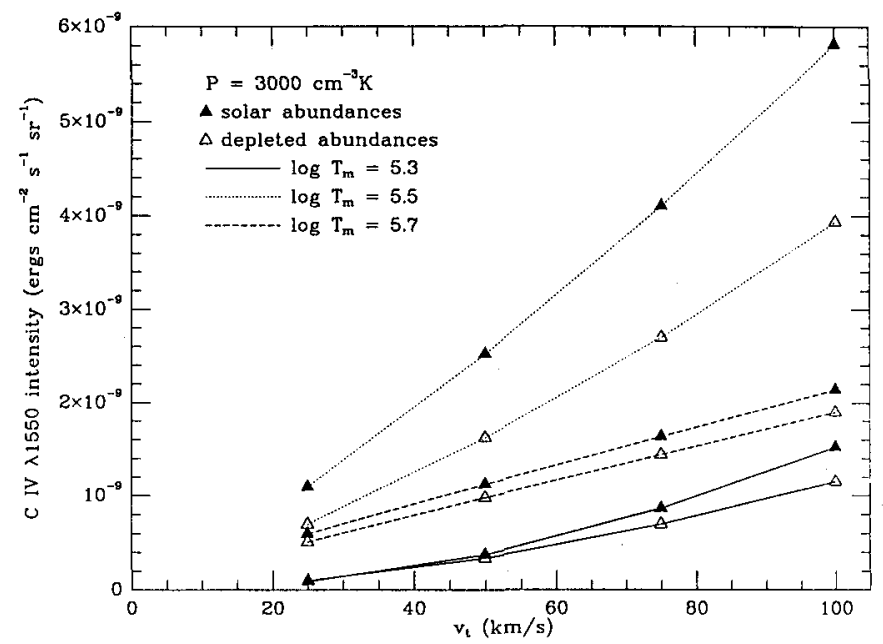

Figure 1: Intensity of C IV $\lambda 1550$ for mixing layers with pressure $n T=3000 \mathrm{~cm}^{-3} \mathrm{~K}$ and three values of $T_{m}$ versus entrainment velocity $v_{t}$. Models with $v_{t}=60-100 \mathrm{~km} \mathrm{~s}^{-1}$ produce the observed ratio C IV $\lambda 1550 /$ O III] $\lambda 1663 \approx 2$.

\section{STOCHASTIC HEATING OF SMALL DUST GRAINS}

In the area of solid-state astrophysics, an exciting development is taking place in the analysis of infrared spectra of small dust grains (radius $a \leq 100 \AA$ ). Because of their low heat capacities, these small grains can be heated far above their equilibrium temperatures by single UV or X-ray photons (Fig. 2). Similar stochastic heating will occur when dust grains are embedded in hot gas (e.g., supernova remnants, clusters of galaxies). In relaxing back to thermal equilibrium, the grains emit infrared radiation characterized by a far-infrared peak produced by the large grains and a mid-and near-infrared plateaul produced by the "flickering" small grains (see Fig. 3).

Dwek $(1986,1987)$ and Yu, Shull, and Voit (1992) have discussed the stochastic heating of dust grains in hot plasma, and Voit (1991) has analyzed the stochastic X-ray heating of grains near quasars and Seyfert galaxies. Although the resulting spectra are similar, it would be valuable to develop models that combine both radiative and collisional heating and identify spectral diagnostics that distinguish between the two processes. It would also be interesting to explore situations in which grain features are driven into emission (e.g., the $10 \mu \mathrm{m}$ and $20 \mu \mathrm{m}$ silicate features or the $3-5 \mu \mathrm{m}$ features associated with polycyclic aromatic hydrocarbons.

\section{THE INTERGALACTIC LYMAN-ALPHA CLOUDS}

I conclude with comments on the probability that knowledge gained from studies of the ISM can be translated to the study of the intergalactic clouds known as the Ly $\alpha$ forest. (Sargent \& Steidel 1990). Recent work in this field suggests the questions: What is the 


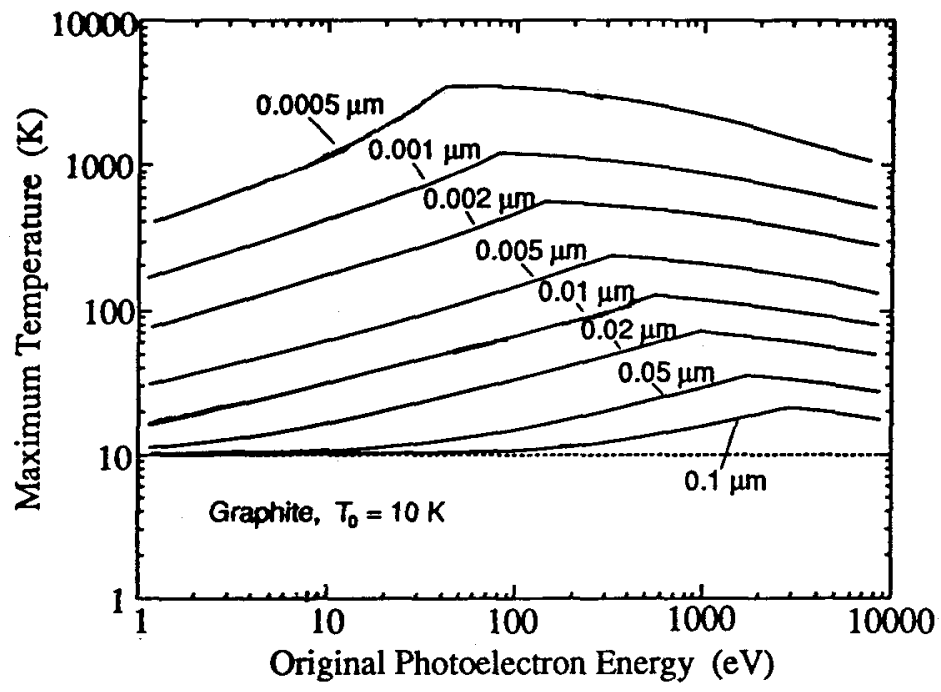

Figure 2: Maximum temperature fluctuations (Voit 1991) of graphite grains of initial temperature $10 \mathrm{~K}$ induced when an X-ray photoelectron travels the entire grain diameter. C'urves, from top to bottom, represent grain radii of $5,10,20,50,100,200,500 \AA, 0.1 \mu \mathrm{m}$, $0.2 \mu \mathrm{m}$, and $0.5 \mu \mathrm{m}$.

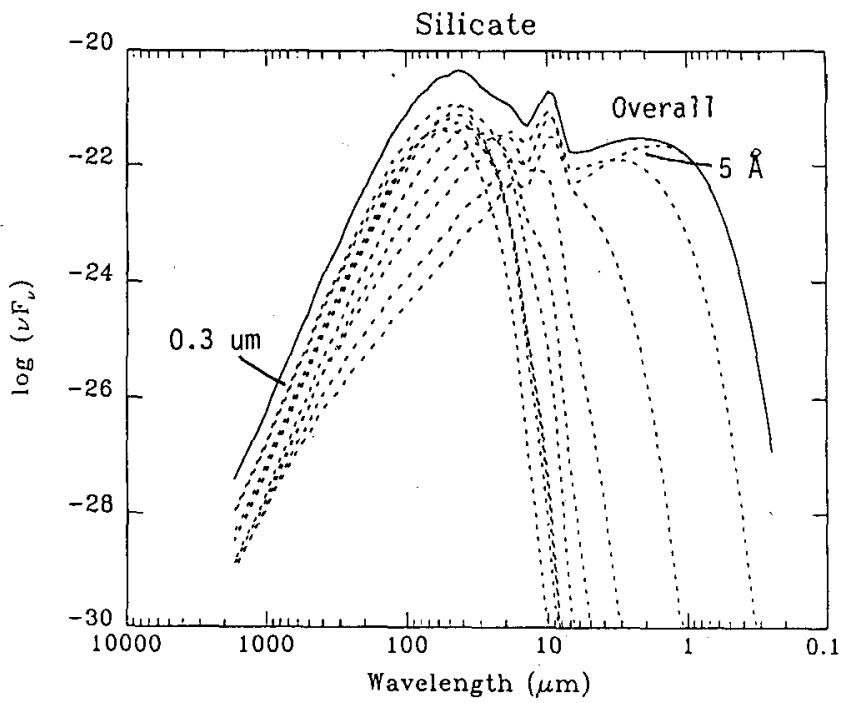

Figure 3: Infrared spectra showing the response of a power-law $\left(a^{-3.5}\right)$ size distribution of silicate grains to stochastic collisional heating by a $10^{7} \mathrm{~K}$ plasma (Yu, Shull, and Voit 1992). The dotted curves illustrate grain sizes ranging from $5 \AA$ to $0.3 \mu \mathrm{m}$. 
source of these clouds? What heats and ionizes them? What confines them ? What is their spatial and temporal evolution?

Several analogies to the ISM come to mind. First, their ionization may be by Lyman continuum from quasars and starburst galaxies, just as interstellar clouds are photoionized by $O$ stars. Second, their source may be "failed galaxies" left over from the era. of galaxy formation, but several pieces of evidence (HST studies toward 3C 273, narrow Ly $\alpha$ line widths) suggest that some clouds may be high-pressure gas resulting from winds from low-luminosity, weakly clustered galaxies, analogous to the action of stellar winds and supernovae in the ISM. Finally, as HST statistics of Ly $\alpha$ absorption clonds at $z \leq 0.2$ accumulate, their spatial distribution and covariance function can be compared with that of low-redshift, low-luminosity galaxies to probe cloud location in galaxy voids. What happens when a Ly $\alpha$ "cloud" encounters hot gas, particularly if it has some motion relative to that medium? Are intergalactic shells and turbulent mixing layers possible? These questions of multiple thermal phases and diverse physical conditions (density, pressure, radiation field) deserve the same scrutiny afforded the ISM.

\section{REFERENCES}

Bahcall, J. N. 1984, ApJ, 276, 169.

Begelman, M. C., \& Fabian, A. 1990, MNRAS, 244, 26P.

Bohlin, R. C., Savage, B. D., \& Drake, J. F. 1978, ApJ, 224, 172.

Cox, D. N. 1990, in The Interstellar Disk-Halo Connection in Galaxies, Kluwer.

Dwek, E. 1986, ApJ, 302, 363.

Dwek, E. 1987, ApJ, 322, 812 .

Edgar, R. J., \& Savage, B. D. 1989, ApJ, 340, 762.

Ferrière, K. M., Zweibel, E. G., \& Shull, J. M. 1988, ApJ, 332, 984.

Jenkins, E. B. 1978a,b, ApJ, 219, 845; ApJ, 220, 107.

Kuijken, K., \& Gilmore, G. 1989a,b,c, MNRAS, 239, 571 \& $605 \& 651$.

Lockman, F. J. 1984, ApJ, 283, 90.

Lockman, F. J., Hobbs, L. M., \& Shull, J. M. 1986, ApJ, 301, 380.

Lyne, A., \& Smith, F. G. 1989, MNRAS, 237, 533.

Martin, C., \& Bowyer, S. 1990, ApJ, 350, 242.

Mathis, J. 1986, ApJ, 301, 423.

Norman, C. 1990, in The Interstellar Disk-Halo Connection in Galaxies, Kluwer.

Rand, R. J., \& Kulkarni, S. R. 1989, ApJ, 343, 760.

Reynolds, R. J. 1982, ApJ, 282, 191.

Reynolds, R. J. 1990, ApJL, 349, L17.

Sargent, W. L. W., \& Steidel, C. C. 1990, in Baryonic Dark Matter, Klıwer.

Savage, B. D., \& Massa, D. 1987, ApJ, 314, 380.

Slavin, J., Shull, J. M., \& Begelman, M. C. 1992, ApJ, submitted.

Van Steenberg, M. E., \& Shull, J. M. 1988, ApJ, 330, 942.

Voit, G. M. 1991, ApJ, 379, 122.

Yu, K., Shull, J. M., \& Voit, G. M. 1992, ApJ, in preparation. 